\title{
Baker-Akhiezer Modules on the Intersections of Shifted Theta Divisors
}

\author{
by
}

\author{
Koji Cho, Andrey Mironov and Atsushi NaKayashiki
}

\begin{abstract}
The restriction, on the spectral variables, of the Baker-Akhiezer (BA) module of a $g$ dimensional principally polarized abelian variety with the non-singular theta divisor to an intersection of shifted theta divisors is studied. It is shown that the restriction to a $k$-dimensional variety becomes a free module over the ring of differential operators in $k$ variables. The remaining $g-k$ derivations define evolution equations for generators of the BA-module. As a corollary new examples of commutative rings of partial differential operators with matrix coefficients and their non-trivial evolution equations are obtained.
\end{abstract}

2010 Mathematics Subject Classification: Primary 14K25; Secondary 14H70.

Keywords: abelian variety, theta function, D-module, commuting differential operators.

\section{$\S 1$. Introduction}

The Baker-Akhiezer (BA) module was introduced in [N1, N2] in order to extend the theory of the BA function due to Krichever $[\mathrm{K}]$ to higher dimensions. It is a geometric counterpart of the $\mathcal{D}$-module generated by the wave operator in Sato's theory of KP-hierarchy and universal Grassmann manifold.

A fundamental example of the BA function is a function on an elliptic curve of the form

$$
\varphi(z ; x)=\frac{\sigma(z+x)}{\sigma(z) \sigma(x)} \exp (-x \zeta(z))
$$

This is a contribution to the special issue "The golden jubilee of algebraic analysis".

Communicated by M. Kashiwara. Received December 24, 2009. Revised February 1 and March 18,2010 .

K. Cho: Department of Mathematics, Kyushu University,

Moto-Oka 744, Nishi-ku, Fukuoka 819-0395, Japan;

e-mail: cho@math.kyushu-u.ac.jp

A. Mironov: Sobolev Institute of Mathematics, Pr. acad. Koptyuga 4, 630090 Novosibirsk, Russia; e-mail: mironov@math.nsc.ru

A. Nakayashiki: Department of Mathematics, Tsuda College,

Tsuda-machi 2-1-1, Kodaira 187-8577, Tokyo, Japan;

e-mail: atsushi@tsuda.ac.jp

(C) 2011 Research Institute for Mathematical Sciences, Kyoto University. All rights reserved. 
where $\sigma(z), \zeta(z)$ are Weierstrass' sigma and zeta functions. The corresponding BA-module is the $\mathcal{D}$-module generated by $\varphi(z ; x)$ :

$$
M=\mathcal{D} \varphi(z ; x)=\sum_{n=0}^{\infty} \mathcal{O} \partial_{x}^{n} \varphi(z ; x),
$$

where $\mathcal{O}$ is a suitable ring of functions such as the convergent power series ring, its quotient field etc. and $\mathcal{D}=\mathcal{O}\left[\partial_{x}\right]$ is the ring of differential operators in $x$ with coefficients in $\mathcal{O}$. It is a rank one free module over $\mathcal{D}$.

Let $A=\mathbb{C}\left[\wp(z), \wp^{\prime}(z)\right]$ be the affine ring of the elliptic curve. An important property of the BA-module is that it is not only a $\mathcal{D}$-module but also an $A$-module. As a consequence $A$ is embedded in $\mathcal{D}$ as a commutative subring.

Similarly, in the case of genus $g$ algebraic curves, the BA-module becomes a $\left(\mathcal{D}_{g}, A\right)$-bimodule, where $\mathcal{D}_{g}=\mathcal{O}\left[\partial_{1}, \ldots, \partial_{g}\right]$ is the ring of differential operators in $g$ variables and $A$ is the affine ring of the curve. It becomes a rank one free module over the subring $\mathcal{D}_{1}=\mathcal{O}\left[\partial_{1}\right]$ of operators in one variable and the affine ring $A$ is embedded in $\mathcal{D}_{1}$. The action of the commuting derivations $\partial_{2}, \ldots, \partial_{g}$ specifies evolution equations of the BA-module, or the deformation of the image of $A$ in $\mathcal{D}$. In this way solutions of integrable nonlinear equations such as KP equation, $\mathrm{KdV}$ equation are constructed $[\mathrm{K}$.

In [N1] the BA-module of a $g$-dimensional principally polarized Abelian variety $(X, \Theta)$ with a non-singular $\Theta$ is studied. It has been proved that the BA-module becomes a free $\mathcal{D}$-module of rank $g$ !, where $\mathcal{D}$ is the ring of differential operators in $g$ variables. Consequently, the affine ring $A$ of $X \backslash \Theta$ is embedded in the ring $\operatorname{Mat}(g !, \mathcal{D})$ of differential operators with coefficients in $g ! \times g !$ matrices. However in this case there is no non-trivial deformation of $A$ in $\operatorname{Mat}(g !, \mathcal{D})$. To have a non-trivial deformation it is necessary to consider the BA-module of polarized subvarieties of $(X, \Theta)$.

We consider an intersection $Y^{k}$ of shifted theta divisors as a subvariety of $X$ and the intersection $Q^{k}$ of it with the theta divisor as a divisor, where $k$ denotes the codimension of $Y^{k}$ in $X$. We show that the restriction of the BA-module of $(X, \Theta)$ to $Y^{k}$ is a free $\mathcal{D}_{g-k}$-module of rank $g$ !, where $\mathcal{D}_{i}$ is the ring of differential operators in $i$ variables. As a by-product we have the embedding of the affine ring of $Y^{k} \backslash Q^{k}$ in $\operatorname{Mat}\left(g !, \mathcal{D}_{g-k}\right)$ and $k$ commuting derivations which specify the deformation of the image of the affine ring.

The simplest case of $g=3$ and $k=1$ is studied in [N2]. The case of intersections of more general divisors is studied in [Mir. Unfortunately the proof of the freeness is incomplete in that paper. Other examples of BA-modules which have non-trivial deformations are studied in $[\mathrm{R}]$. 
The plan of the paper is as follows. In Section 2 the definition of BA-modules and the main result are given. The combinatorial properties of the restriction of the BA-module is studied in Section 3. It is shown that the character of the associated graded module of the BA-module coincides with that of the free module. This means that as far as the dimension is concerned the restriction of the BA-module becomes a free module. In Section 4 the proof of the main theorem is given based on the result of Section 3 .

\section{§2. Results}

Let $\Omega$ be a point of the Siegel upper half space of degree $g, X$ the corresponding principally polarized Abelian variety

$$
X=\mathbb{C}^{g} / \Gamma, \quad \Gamma=\mathbb{Z}^{g}+\Omega \mathbb{Z}^{g},
$$

$\theta_{a, b}(z)$ Riemann's theta function with characteristic $(a, b), a, b \in \mathbb{R}^{g}$,

$$
\theta_{a, b}(z)=\theta_{a, b}(z, \Omega)=\sum_{n \in \mathbb{Z}^{g}} \exp \left(\pi i^{t}(n+a) \Omega(n+a)+2 \pi i^{t}(n+a)(z+b)\right),
$$

and $\Theta$ the theta divisor on $X$ defined by the zero set of $\theta(z)=\theta_{0,0}(z)$. For $c \in \mathbb{C}^{g}, \mathcal{L}_{c}$ denotes the holomorphic flat line bundle on $X$ which has $\theta(z+c) / \theta(z)$ as a meromorphic section. A meromorphic section of $\mathcal{L}_{c}$ can be considered as a meromorphic function $f(z)$ on $\mathbb{C}^{g}$ which satisfies

$$
f(z+m+\Omega n)=\exp \left(-2 \pi i^{t} n c\right) f(z), \quad m, n \in \mathbb{Z}^{g} .
$$

We denote by $L_{c}(n)$ the space of meromorphic sections of $\mathcal{L}_{c}$ whose poles are only on $\Theta$ of order at most $n$. It is known M2 that $\operatorname{dim} L_{c}(n)=n^{g}$ and a linear basis is given by the functions $f_{n, a}(z+c / n) / \theta(z)^{n}, a \in \mathbb{Z}^{g} / n \mathbb{Z}^{g}$, where

$$
f_{n, a}(z)=\theta_{a / n, 0}(n z, n \Omega) .
$$

We define

$$
L_{c}=\bigcup_{n=0}^{\infty} L_{c}(n)
$$

The subspaces $L_{c}(n)$ define an increasing filtration of $L_{c}$.

Set

$$
\zeta_{i}(z)=\frac{\partial}{\partial z_{i}} \log \theta(z)
$$

It satisfies, for $m, n \in \mathbb{Z}^{g}$,

$$
\zeta_{j}(z+m+\Omega n)=\zeta_{j}(z)-2 \pi i n_{j} .
$$


Let $\mathcal{O}$ be the convergent power series ring in $x=\left(x_{1}, \ldots, x_{g}\right), \mathcal{K}$ its quotient field, $\partial_{x_{i}}=\partial / \partial x_{i}, \mathcal{D}=\mathcal{K}\left[\partial_{x_{1}}, \ldots, \partial_{x_{g}}\right]$ the ring of differential operators with coefficients in $\mathcal{K}, \mathcal{D}(n)=\left\{\sum_{|\alpha|<n} a_{\alpha} \partial_{x}^{\alpha} \in \mathcal{D}\right\}$ the differential operators of order at most $n$ and gr $\mathcal{D}=\bigoplus \mathcal{D}(n) / \mathcal{D}(n-1)$ the commutative ring of principal symbols.

In general, for a module with an increasing filtration $M=\bigcup_{n} M(n)$, the associated graded module is defined by

$$
\operatorname{gr} M=\bigoplus_{n} \operatorname{gr}_{n} M, \quad \operatorname{gr}_{n} M=M(n) / M(n-1) .
$$

Let us consider the spaces of functions of the form:

$$
\begin{aligned}
M_{c}(n) & =\sum_{a \in \mathbb{Z}^{g} / n \mathbb{Z}^{g}} \mathcal{K} \frac{f_{n, a}\left(z+\frac{c+x}{n}\right)}{\theta(z)^{n}} \exp \left(-\sum_{i=1}^{g} x_{i} \zeta_{i}(z)\right), \\
M_{c} & =\bigcup_{n=0}^{\infty} M_{c}(n) .
\end{aligned}
$$

The space $M_{c}(n)$ is an $n^{g}$-dimensional vector space over $\mathcal{K}$ and the subspaces $\left\{M_{c}(n)\right\}$ specify an increasing filtration of $M_{c}$. Then, for $c \notin \Gamma$,

$$
\operatorname{dim}_{\mathcal{K}} \operatorname{gr}_{n} M_{c}=\operatorname{dim}_{\mathcal{K}} \operatorname{gr}_{n} L_{c}=n^{g}-(n-1)^{g}, \quad n \geq 1 .
$$

As functions of $z$ variables, elements of $M_{c}$ satisfy the equation (2.1). The differentiation in $x_{i}$ preserves this equation and satisfies

$$
\partial_{x_{i}} M_{c}(n) \subset M_{c}(n+1) .
$$

Thus $M_{c}$ becomes a $\mathcal{D}$-module and gr $M_{c}$ becomes a gr $\mathcal{D}$-module. The $\mathcal{D}$-module $M_{c}$ is introduced in [N1] and called the Baker-Akhiezer (BA) module of $(X, \Theta)$.

Let $A$ be the affine ring of $X \backslash \Theta$. Analytically it is described as

$$
A=\left\{\frac{F(z)}{\theta(z)^{n}} \mid F(z) \text { is holomorphic on } \mathbb{C}^{g}, \frac{F}{\theta^{n}}(z+\gamma)=\frac{F}{\theta^{n}}(z) \text { for any } \gamma \in \Gamma\right\} .
$$

If $f(z)$ satisfies (2.1) and $a(z) \in A$ then $f(z) a(z)$ satisfies (2.1). Therefore $L_{c}$ is an $A$-module. Consequently, $M_{c}$ becomes an $A$-module whose action obviously commutes with that of $\mathcal{D}$.

Remark. In [N1] the BA-module is defined globally on the dual abelian variety $\widehat{X}$ of $X$ as a sheaf of $\mathcal{D}_{\widehat{X}}$-modules by the Fourier-Mukai transform, $M_{c}$ being a scalar extension of the stalk at the point specified by $c$. 
Let $r_{i}, i \geq 1$, be integers defined by

$$
r_{i}=i^{g}-(i-1)^{g}-\sum_{j=1}^{i-1} r_{j}\left(\begin{array}{c}
g+i-j-1 \\
g-1
\end{array}\right) .
$$

They satisfy $r_{1}=1, r_{i}=r_{g+1-i}, r_{i}=0$ for $i>g$ and $\sum_{i=1}^{g} r_{i}=g !$.

The following theorem is proved in $\mathrm{N} 1$.

Theorem 2.1 ([N1]). Suppose that $\Theta$ is non-singular and $c \notin \Gamma$. Then $\operatorname{gr} M_{c}$ is a free gr $\mathcal{D}$-module of rank $g$ ! and $M_{c}$ is a free $\mathcal{D}$-module of rank $g$ !. More precisely

$$
\begin{aligned}
\operatorname{gr} M_{c} & =\bigoplus_{i=1}^{g} \bigoplus_{j=1}^{r_{i}}(\operatorname{gr} \mathcal{D}) \psi_{i j}, \quad \psi_{i j} \in \operatorname{gr}_{i} M_{c} \\
M_{c} & =\bigoplus_{i=1}^{g} \bigoplus_{j=1}^{r_{i}} \mathcal{D} \phi_{i j}, \quad \phi_{i j} \in M_{c}(i)
\end{aligned}
$$

where $\psi_{i j}$ is the projection of $\phi_{i j}$ in $\operatorname{gr}_{i} M_{c}$.

Let $\Phi$ be the column vector of dimension $g$ ! whose components consist of the functions $\phi_{i j}$ and $\operatorname{Mat}(m, \mathcal{D})$ the ring of $m \times m$ matrices with components in $\mathcal{D}$. Since $M_{c}$ is an $A$-module, for $a \in A$, there exists a unique element $\ell(a) \in$ $\operatorname{Mat}(g !, \mathcal{D})$ such that

$$
a \Phi=\ell(a) \Phi .
$$

This defines an embedding of $A$ into $\operatorname{Mat}(g !, \mathcal{D})$ as a ring. Thus $A$ is realized as a commutative subring of the ring of differential operators in $g$ variables with matrix coefficients.

In this paper we shall extend Theorem 2.1 to the BA-modules on intersections of shifted theta divisors.

For $a \in \mathbb{C}^{g}$ we set

$$
\Theta_{a}=\{\theta(z-a)=0\} \subset X
$$

Take $a_{1}, \ldots, a_{g-1} \in \mathbb{C}^{g}$. Beginning from $\left(Y^{0}, Q^{0}\right)=(X, \Theta)$ we define $\left(Y^{k}, Q^{k}\right)$ for $k \geq 1$ by

$$
\begin{aligned}
& Y^{k}=\Theta_{a_{1}} \cap \cdots \cap \Theta_{a_{k}}, \\
& Q^{k}=Y^{k} \cap \Theta .
\end{aligned}
$$

We assume that, for any $k<g, \Theta_{a_{1}}, \ldots, \Theta_{a_{k}}$ intersect transversally and so do $\Theta, \Theta_{a_{1}}, \ldots, \Theta_{a_{k}}$. This means in particular that $Y^{k}$ and $Q^{k}$ are non-singular subvarieties of $X$ of dimensions $g-k$ and $g-k-1$ respectively. It can be shown that for generic $a_{1}, \ldots, a_{g-1}$ the assumption is satisfied. 
We denote the restriction of $\mathcal{L}_{c}$ to $Y^{k}$ by the same symbol for simplicity. Let $\mathcal{L}_{c}\left(n Q^{k}\right)$ be the sheaf of germs of meromorphic sections of $\mathcal{L}_{c}$ on $Y^{k}$ with poles only on $Q^{k}$ of order at most $n$. We set

$$
L_{c}^{k}(n)=H^{0}\left(Y^{k}, \mathcal{L}_{c}\left(n Q^{k}\right)\right) .
$$

We define the space $\tilde{M}_{c}^{k}(n)$ as the set of functions of the form

$$
f(z ; x) \exp \left(-\left.\sum_{i=1}^{g} x_{i} \zeta_{i}(z)\right|_{Y^{k}}\right),
$$

where $f(z ; x)$ satisfies the following conditions.

There is an open neighborhood $U$ of $0 \in \mathbb{C}^{g}$, which can depend on $f$, with the following properties.

(i) For each $x \in U, f(z ; x)$ belongs to $L_{c+x}^{k}(n)$ as a function of $z$.

(ii) As a function of $x, f(z ; x)$ is analytic on $U$.

It is obvious that $\tilde{M}_{c}^{k}(n)$ is an $\mathcal{O}$-module.

Lemma 2.2. Let $k$ satisfy $0 \leq k \leq g-1$. Suppose that $c+\sum_{i \in I} a_{i} \notin \Gamma$ for any subset $I$ of $\{1, \ldots, k\}$. Then

(i) $H^{i}\left(Y^{k}, \mathcal{L}_{c}\left(n Q^{k}\right)\right)=0, i \neq 0, g-k, n \in \mathbb{Z}$.

(ii) The restriction map $L_{c}^{k-1}(n) \rightarrow L_{c}^{k}(n)$ is surjective for any $n \in \mathbb{Z}$.

Proof. We have the following exact sequence and isomorphism:

$$
\begin{aligned}
& 0 \rightarrow \mathcal{L}_{c}\left(n Q^{k-1}-Y^{k}\right) \rightarrow \mathcal{L}_{c}\left(n Q^{k-1}\right) \rightarrow \mathcal{L}_{c}\left(n Q^{k}\right) \rightarrow 0 \\
& \mathcal{L}_{c}\left(n Q^{k-1}-Y^{k}\right) \simeq \mathcal{L}_{c+a_{k}}\left((n-1) Q^{k-1}\right)
\end{aligned}
$$

The isomorphism 2.4 follows from $\mathcal{O}_{X}\left(-\Theta_{a}\right) \simeq \mathcal{O}_{X}(-\Theta) \otimes \mathcal{L}_{a}$. Assertion (i) can be proved by induction on $k$ using the cohomology sequence of 2.3 and the vanishing M1

$$
H^{i}\left(X, \mathcal{L}_{c}(n \Theta)\right)=0
$$

for $i \geq 1, n \geq 1$ or $i \geq 0, n=0$ or $i \neq g, n<0$. Assertion (ii) follows from the cohomology sequence of $(2.3)$ and (i).

Lemma 2.3. Assume the same conditions as in Lemma 2.2. Then $\operatorname{dim} L_{c}^{k}(n)$ does not depend on $c$ and satisfies

$$
\operatorname{dim} L_{c}^{k}(n)=\operatorname{dim} L_{c}^{k-1}(n)-\operatorname{dim} L_{c}^{k-1}(n-1) .
$$


Proof. The lemma can easily be proved by induction on $k$ using the cohomology sequence of 2.3), the isomorphism (2.4), Lemma 2.2(i) and $\operatorname{dim} L_{c}^{0}(n)=n^{g}$ for $n \geq 0, c \notin \Gamma$.

\section{Example 2.4.}

$$
\begin{aligned}
& \operatorname{dim} L_{c}^{1}(n)=n^{g}-(n-1)^{g} \quad \text { for } n \geq 1, \\
& \operatorname{dim} L_{c}^{2}(n)=n^{g}-2(n-1)^{g}+(n-2)^{g} \quad \text { for } n \geq 2 \quad \text { and } \quad \operatorname{dim} L_{c}^{2}(1)=1 .
\end{aligned}
$$

Lemma 2.5 (M1]). Assume the same conditions as in Lemma 2.2. Then $\tilde{M}_{c}^{k}$ is a free $\mathcal{O}$-module of rank $\operatorname{dim} L_{c}^{k}(n)$.

Proof. We take some basis $\left\{f_{i}(z)\right\}$ of $L_{c}^{k}(n)$ and lift it to $\left\{f_{i}(z ; x)\right\}$ such that the conditions (i), (ii) of $\tilde{M}_{c}^{k}(n)$ are satisfied and $f_{i}(z ; 0)=f_{i}(z)$. This gives an $\mathcal{O}$ free basis of $\tilde{M}_{c}^{k}(n)$. Such analytic lift can be constructed among the functions $\left\{f_{n, a}\left(z+\frac{c+x}{n}\right)\right\}$ restricted to $Y^{k}$ since the restriction map $L_{c+x}^{0}(n) \rightarrow L_{c+x}^{k}(n)$ is surjective for $x$ sufficiently close to $0 \in \mathbb{C}^{g}$ by Lemma 2.2(ii).

We set

$$
M_{c}^{k}(n)=\mathcal{K} \otimes_{\mathcal{O}} \tilde{M}_{c}^{k}(n), \quad M_{c}^{k}=\bigcup M_{c}^{k}(n) .
$$

The set of subspaces $\left\{M_{c}^{k}(n)\right\}$ defines an increasing filtration on $M_{c}^{k}$.

Let $\pi_{k}: M_{c}^{k} \rightarrow M_{c}^{k+1}$ be the restriction map. It is surjective for $k \geq 0$ as shown above. In particular $\pi_{0 k}:=\pi_{k-1} \cdots \pi_{0}: M_{c} \rightarrow M_{c}^{k}$ is surjective. Thus $M_{c}^{k}$ can be directly described as the restriction of $M_{c}$ to $Y^{k}$ with respect to the $z$ variables:

$$
M_{c}^{k}=\left.M_{c}\right|_{Y^{k}} .
$$

It is obvious that the restriction in $z$ variables commutes with the action of $\partial_{x_{i}}$. Therefore $M_{c}^{k}$ becomes a $\mathcal{D}$-module. Moreover the action of $\partial_{x_{i}}$ satisfies $\partial_{x_{i}} M_{c}^{k}(n)$ $\subset M_{c}^{k}(n+1)$. Thus gr $M_{c}^{k}$ becomes a gr $\mathcal{D}$-module.

The main result of this paper is

Theorem 2.6. Suppose that $\Theta$ is non-singular and $c+\sum_{i \in I} a_{i} \notin \Gamma$ for any subset $I$ of $\{1, \ldots, g-1\}$. Then there exists a set of linearly independent vector fields $D_{i}=\sum_{j=1}^{g} c_{i j} \partial_{x_{j}}, c_{i j}$ being constants, such that the following properties are valid.

(i) Let $\mathcal{D}_{i}=\mathcal{K}\left[D_{1}, \ldots, D_{i}\right]$. Then $M_{c}^{k}$ is a free $\mathcal{D}_{g-k}$-module of rank $g$ !. More precisely it is of the form

$$
M_{c}^{k}=\bigoplus_{i=1}^{g} \bigoplus_{j=1}^{r_{i}} \mathcal{D}_{g-k} \phi_{i j}^{k}, \quad \phi_{i j}^{k} \in M_{c}^{k}(i) .
$$


(ii) The module gr $M_{c}^{k}$ is a free gr $\mathcal{D}_{g-k}$-module of rank $g$ !. More precisely it is of the form

$$
\operatorname{gr} M_{c}^{k}=\bigoplus_{i=1}^{g} \bigoplus_{j=1}^{r_{i}}\left(\operatorname{gr} \mathcal{D}_{g-k}\right) \psi_{i j}^{k}, \quad \psi_{i j}^{k} \in \operatorname{gr}_{i} M_{c}^{k},
$$

where $\psi_{i j}^{k}$ is the image of $\phi_{i j}^{k}$ in $\operatorname{gr}_{i} M_{c}^{k}$ and the filtration of $\mathcal{D}_{g-k}$ is specified by $\mathcal{D}_{k}(n)=\mathcal{D}_{k} \cap \mathcal{D}(n)$.

Let $\Phi^{k}$ be the column vector whose components consist of the functions $\phi_{i j}^{k}$ and $A^{k}$ be the affine ring of $Y^{k} \backslash Q^{k}$. We have $\Phi^{0}=\Phi, A^{0}=A$. The ring $A^{k}$ acts on $M_{c}^{k}$. Thus for any $a \in A^{k}$ there exists a unique operator $\ell^{k}(a) \in \operatorname{Mat}\left(g !, \mathcal{D}_{g-k}\right)$ such that

$$
a \Phi^{k}=\ell^{k}(a) \Phi^{k} .
$$

This defines an embedding of $A^{k}$ in $\operatorname{Mat}\left(g !, \mathcal{D}_{g-k}\right)$. Since $M_{c}^{k}$ is a $\mathcal{D}$-module and $\mathcal{D}=\mathcal{D}_{g}$, for $D_{i}$ with $g-k+1 \leq i \leq g$, there exists a unique operator $B_{i}^{k} \in$ $\operatorname{Mat}\left(g !, \mathcal{D}_{g-k}\right)$ such that

$$
D_{i} \Phi^{k}=B_{i}^{k} \Phi^{k}
$$

Those operators satisfy, for any $a, b, i, j$,

$$
\left[\ell^{k}(a), \ell^{k}(b)\right]=0, \quad\left[D_{i}-B_{i}^{k}, D_{j}-B_{j}^{k}\right]=0, \quad\left[D_{i}-B_{i}^{k}, \ell^{k}(a)\right]=0 .
$$

This system of equations is an analogue of the Zakharov-Shabat equations $[\mathrm{K}$.

\section{$\S 3$. Combinatorial freeness}

For a graded $\mathcal{K}$-vector space $V=\bigoplus_{n \in \mathbb{Z}} V_{n}$ such that each $V_{n}$ is finite-dimensional we define the character $\operatorname{ch} V$ by

$$
\operatorname{ch} V=\sum\left(\operatorname{dim}_{\mathcal{K}} V_{n}\right) t^{n} .
$$

Obviously

We have

$$
\operatorname{chgr} \mathcal{D}_{j}=\frac{1}{(1-t)^{j}}
$$

$$
\operatorname{chgr} M_{c}^{0}=\sum_{n=1}^{\infty}\left(n^{g}-(n-1)^{g}\right) t^{n}=(1-t)\left(t \frac{d}{d t}\right)^{g}(1-t)^{-1} .
$$

Let $\left\{\psi_{i j}\right\}$ be a gr $\mathcal{D}$-free basis of gr $M_{c}$ as in Theorem 2.1 and $F=\bigoplus \mathcal{K} \psi_{i j}$ the subspace of gr $M_{c}$. By Theorem 2.1, gr $M_{c} \simeq\left(\operatorname{gr} \mathcal{D}_{g}\right) \otimes F$. The module $F$ naturally inherits a grading from gr $M_{c}$. Then

$$
\operatorname{chgr} M_{c}=\left(\operatorname{chgr} \mathcal{D}_{g}\right) \cdot \operatorname{gr} F=(1-t)^{-g} \sum_{i=1}^{g} r_{i} t^{i} .
$$


Example 3.1. For $g=1,2,3,4$, ch gr $M_{c}$ is given by

$$
\frac{t}{1-t}, \quad \frac{t+t^{2}}{(1-t)^{2}}, \quad \frac{t+4 t^{2}+t^{3}}{(1-t)^{3}}, \quad \frac{t+11 t^{2}+11 t^{3}+t^{4}}{(1-t)^{4}} .
$$

Lemma 3.2. Assume the same conditions for $c, a_{1}, \ldots, a_{g-1}$ as in Theorem 2.6. Then

(i) $\operatorname{dim}_{\mathcal{K}} \operatorname{gr}_{n} M_{c}^{j+1}=\operatorname{dim}_{\mathcal{K}} \operatorname{gr}_{n} M_{c}^{j}-\operatorname{dim}_{\mathcal{K}} \operatorname{gr}_{n-1} M_{c}^{j}$.

(ii) $\operatorname{chgr} M_{c}^{j+1}=(1-t) \operatorname{chgr} M_{c}^{j}$.

Proof. Asertion (ii) follows from (i), and (i) follows from Lemmas 2.3 and 2.5

By Lemma 3.2 we have

$$
\operatorname{chgr} M_{c}^{j}=(1-t)^{j} \operatorname{chgr} M_{c}=\left(\operatorname{ch} \mathcal{D}_{g-j}\right) \cdot \operatorname{ch} F .
$$

\section{$\S 4$. Proof of Theorem 2.6}

Notice that (i) of Theorem 2.6 follows from (ii).

We shall prove

Proposition 4.1. Assume the same conditions as in Theorem 2.6. Set $y^{(0)}=$ $\left(y_{1}^{(0)}, \ldots, y_{g}^{(0)}\right)=\left(x_{1}, \ldots, x_{g}\right)$. Then, for each $k \geq 1$, there exist a coordinate $y^{(k)}=$ $\left(y_{1}^{(k)}, \ldots, y_{g-k+1}^{(k)}\right)$, a linear change of coordinates from $\left(y_{1}^{(k-1)}, \ldots, y_{g-k+1}^{(k-1)}\right)$ to $y^{(k)}$ and $\psi_{i j}^{k} \in \operatorname{gr}_{i} M_{c}^{k}, 1 \leq i \leq g, 1 \leq j \leq r_{i}$ such that the following properties hold. Let $\mathcal{D}_{g-k}=\mathcal{K}\left[\partial_{y_{1}^{(k)}}, \ldots, \partial_{y_{g-k}^{(k)}}\right], \mathcal{D}_{g-k}(n)=\mathcal{D}_{g-k} \cap \mathcal{D}(n)$ and $\xi_{i}^{(k)}$ the image of $\partial_{y_{i}^{(k)}}$ in $\operatorname{gr}_{1} \mathcal{D}$. Then

$$
\begin{aligned}
& \xi_{g-k+1}^{(k)} \psi_{i j}^{k} \in \sum_{i^{\prime} j^{\prime}}\left(\operatorname{gr} \mathcal{D}_{g-k}\right) \psi_{i^{\prime} j^{\prime}}^{k}, \\
& \operatorname{gr} M_{c}^{k}=\bigoplus_{i=1}^{g} \bigoplus_{j=1}^{r_{i}}\left(\operatorname{gr} \mathcal{D}_{g-k}\right) \psi_{i j}^{k} .
\end{aligned}
$$

If we define $D_{k}=\partial_{y_{k}^{(g+1-k)}}$ for $2 \leq k \leq g$ and $D_{1}=\partial_{y_{1}^{(g-1)}}$, then Theorem 2.6(ii) follows from this proposition.

We prove Proposition 4.1 by induction on $k$, where the case $k=0$ is established by Theorem 2.1. We assume that the assertion is valid for $k$ if $c+\sum_{i \in I} a_{i} \notin \Gamma$ for any subset $I$ of $\{1, \ldots, k\}$. We shall prove that it is true for $k+1$ if $c+\sum_{i \in I} a_{i}$ $\notin \Gamma$ for any subset $I$ of $\{1, \ldots, k+1\}$.

Let us set

$$
\tilde{\psi}_{i j}^{k+1}=\left.\psi_{i j}^{k}\right|_{Y^{k+1}}
$$


Lemma 4.2. For each $(i, j)$ there exist a non-zero element $P_{i j} \in \operatorname{gr}_{N^{i j}} \mathcal{D}_{g-k}$ for some $N^{i j} \geq 0$ and a linear change of coordinates from $\left(y_{1}^{(k)}, \ldots, y_{g-k}^{(k)}\right)$ to $\left(y_{1}^{(k+1)}, \ldots, y_{g-k}^{(k+1)}\right)$ such that the following properties hold:

(i) $P_{i j} \tilde{\psi}_{i j}^{k+1}=0$ in $\operatorname{gr} M_{c}^{k+1}$.

(ii) Let $\xi_{i}=\xi_{i}^{(k+1)}$. Then $P_{i j}$ is of the form

$$
P_{i j}=\xi_{g-k}^{N^{i j}}+\sum_{|\alpha|=N^{i j}, \alpha_{g-k}<N^{i j}} a_{i j ; \alpha} \xi_{1}^{\alpha_{1}} \cdots \xi_{g-k}^{\alpha_{g-k}},
$$

where $\alpha=\left(\alpha_{1}, \ldots, \alpha_{g-k}\right),|\alpha|=\sum_{i=1}^{g-k} \alpha_{i}$.

Proof. (i) By 3.1 the dimension of $\operatorname{gr}_{n} M_{c}^{k+1}$ is a polynomial in $n$ of degree $g-k-2$ for sufficiently large $n$. If there are no non-trivial linear relations among $\xi_{1}^{\alpha_{1}} \cdots \xi_{g-k}^{\alpha_{g-k}} \tilde{\psi}_{i j}^{k+1}, \sum \alpha_{l}=n-i$ for any $n$, then $\operatorname{dim}_{\mathcal{K}}\left(\left(\operatorname{gr}_{n-i} \mathcal{D}_{g-k}\right) \tilde{\psi}_{i j}^{k+1}\right)=$ $\operatorname{dim}_{\mathcal{K}} \operatorname{gr}_{n-i} \mathcal{D}_{g-k}$ and it is a polynomial in $n$ of degree $g-k-1$ for sufficiently large $n$. Thus there should be a relation as in the assertion.

(ii) Let us write

$$
P_{i j}=\sum_{\sum \alpha_{l}=N^{i j}} q_{\alpha_{1}, \ldots, \alpha_{g-k}} \xi_{1}^{\alpha_{1}} \cdots \xi_{g-k}^{\alpha_{g-k}},
$$

Renaming the variables if necessary one can assume that $q_{\alpha_{1}, \ldots, \alpha_{g-k}} \neq 0$ for some $\left(\alpha_{1}, \ldots, \alpha_{g-k}\right)$ with $\alpha_{g-k}$. If $q_{0, \ldots, 0, N^{i j}} \neq 0$ then we get the desired element by dividing $P_{i j}$ by $q_{0, \ldots, 0, N^{i j}} \neq 0$. If this is not the case, we consider the change of variables of the form

$$
\xi_{i}^{(k)}=\sum_{l=i}^{g-k} c_{i, l} \xi_{l}^{(k+1)} .
$$

Let $c_{i}=c_{i, g-k}$. Then in the resulting expression of $P_{i j}$ the coefficient of $\left(\xi_{g-k}^{(k+1)}\right)^{N^{i j}}$ is

$$
\sum_{|\alpha|=N^{i j}} c_{1}^{\alpha_{1}} \cdots c_{g-k}^{\alpha_{g-k}} q_{\alpha_{1}, \ldots, \alpha_{g-k}}
$$

This is a non-zero homogeneous polynomial in $c_{1}, \ldots, c_{g-k}$. Thus it is non-zero on a non-empty open subset of $\mathbb{C}^{g-k}$. Take a point in this set, make a change of coordinates and dividing $P_{i j}$ by 4.1 we get the desired result.

Let

$$
\operatorname{gr} \pi_{k}: \operatorname{gr} M_{c}^{k} \rightarrow \operatorname{gr} M_{c}^{k+1}
$$

be the restriction map induced by $\pi_{k}$ and $K^{k}=\bigoplus K_{n}^{k}$ the kernel of gr $\pi_{k}$. Since gr $\pi_{k}$ is a homomorphism of gr $\mathcal{D}$-modules, $K^{k}$ is a gr $\mathcal{D}$-submodule of gr $M_{c}^{k}$. We 
denote by $\tilde{K}^{k}=\bigoplus \tilde{K}_{n}^{k}$ the gr $\mathcal{D}$-module obtained from $K^{k}$ by shifting the grading by -1 , that is, $\tilde{K}_{n}^{k}=K_{n+1}^{k}$.

Lemma 4.3. The map

$$
\operatorname{gr}_{n} M_{c+a_{k+1}}^{k} \rightarrow K_{n+1}^{k}, \quad \phi(z) \mapsto \frac{\theta\left(z-a_{k+1}\right)}{\theta(z)} \phi(z),
$$

gives an isomorphism of gr $M_{c+a_{k+1}}^{k}$ and $\tilde{K}^{k}$ as gr $\mathcal{D}_{g-k}$-modules.

Proof. We can assume $g-k \geq 2$. Using Lemma 2.2(i), 2.4 and the cohomology sequence of 2.3 we have

$$
\operatorname{Ker}\left(\left.\pi_{k}\right|_{M_{c}^{k}(n)}\right)=\left.\frac{\theta\left(z-a_{k+1}\right)}{\theta(z)}\right|_{Y^{k}} M_{c+a_{k+1}}^{k}(n-1) .
$$

Let $\operatorname{gr}_{n}\left(\left.\mathcal{L}_{c}\left(-m Y^{k+1}\right)\right|_{Y^{k}}\right)$ be the sheaf on $Y^{k}$ defined by the exact sequence

$$
\begin{aligned}
0 \rightarrow \mathcal{L}_{c}\left((n-1) Q^{k}-m Y^{k+1}\right) & \rightarrow \mathcal{L}_{c}\left(n Q^{k}-m Y^{k+1}\right) \\
& \rightarrow \operatorname{gr}_{n}\left(\left.\mathcal{L}_{c}\left(-m Y^{k+1}\right)\right|_{Y^{k}}\right) \rightarrow 0 .
\end{aligned}
$$

Then one can easily verify that the following sequence is exact:

$$
0 \rightarrow \operatorname{gr}_{n}\left(\left.\mathcal{L}_{c}\left(-Y^{k+1}\right)\right|_{Y^{k}}\right) \rightarrow \operatorname{gr}_{n}\left(\left.\mathcal{L}_{c}\right|_{Y^{k}}\right) \rightarrow \operatorname{gr}_{n}\left(\left.\mathcal{L}_{c}\right|_{Y^{k+1}}\right) \rightarrow 0 .
$$

By the cohomology sequence of 4.4 with $m=0$ and Lemma 2.2(i), the natural map

$$
\operatorname{gr}_{n} L_{c}^{k}:=L_{c}^{k}(n) / L_{c}^{k}(n-1) \rightarrow H^{0}\left(Y^{k}, \operatorname{gr}_{n}\left(\left.\mathcal{L}_{c}\right|_{Y^{k}}\right)\right)
$$

is always injective and becomes isomorphic if $g-k \geq 2$. Then we have

$$
\begin{aligned}
\operatorname{Ker}\left(\operatorname{gr}_{n} L_{c}^{k} \rightarrow \operatorname{gr}_{n} L_{c}^{k+1}\right) & \simeq H^{0}\left(Y^{k}, \operatorname{gr}_{n}\left(\left.\mathcal{L}_{c}\left(-Y^{k+1}\right)\right|_{Y^{k}}\right)\right) \\
& \simeq \frac{H^{0}\left(Y^{k}, \mathcal{L}_{c}\left(n Q^{k}-Y^{k+1}\right)\right)}{H^{0}\left(Y^{k}, \mathcal{L}_{c}\left((n-1) Q^{k}-Y^{k+1}\right)\right)} \\
& \left.\simeq \frac{\theta\left(z-a_{k+1}\right)}{\theta(z)}\right|_{Y^{k}} \operatorname{gr}_{n-1} L_{c+a_{k+1}}^{k},
\end{aligned}
$$

where we use the cohomology sequences of 4.5), 4.4, Lemma 2.2(i), 2.4, 44.3).

If $c+a_{k+1}+\sum_{i \in I} a_{i} \notin \Gamma$ for any subset $I$ of $\{1, \ldots, k\}$ the induction hypothesis can be applied to $M_{c+a_{k+1}}^{k}$. By the lemma and the inductive assumption, $K^{k}$ is 
described as

$$
\begin{aligned}
K^{k} & =\bigoplus_{i=1}^{g} \bigoplus_{j=1}^{r_{i}}\left(\operatorname{gr} \mathcal{D}_{g-k}\right) \varphi_{i j} \\
\varphi_{i j} & =\left.\frac{\theta\left(z-a_{k+1}\right)}{\theta(z)} \psi_{i j}^{k}\right|_{c \rightarrow c+a_{k+1}} \in K_{i+1}^{k}
\end{aligned}
$$

Since $\varphi_{i j} \in \operatorname{gr}_{i+1} M_{c}^{k}$, it can be written as a linear combination of $\left\{\psi_{i j}^{k}\right\}$ with coefficients in gr $\mathcal{D}_{g-k}$ as

$$
\varphi_{i j}=\sum Q_{i j: i^{\prime} j^{\prime}} \psi_{i^{\prime} j^{\prime}}^{k}, \quad Q_{i j: i^{\prime} j^{\prime}}=\sum_{|\alpha|+i^{\prime}=i+1} q_{i j ; i^{\prime} j^{\prime}}^{\alpha} \xi^{\alpha}
$$

By Lemma 4.2 (i) we have $P_{i j} \psi_{i j}^{k} \in K_{i+N^{i j}}^{k}$. Thus it can be written as a linear combination of $\left\{\varphi_{i j}\right\}$ with coefficients in gr $\mathcal{D}_{g-k}$ as

$$
P_{i j} \psi_{i j}^{k}=\sum R_{i j ; i^{\prime} j^{\prime}} \varphi_{i^{\prime} j^{\prime}}, \quad R_{i j ; i^{\prime} j^{\prime}}=\sum_{|\alpha|+i^{\prime}+1=i+N^{i j}} r_{i j ; i^{\prime} j^{\prime}}^{\alpha} \xi^{\alpha} .
$$

Composing these relations we get

$$
P_{i j} \psi_{i j}^{k}=\sum R_{i j ; i^{\prime} j^{\prime}} Q_{i^{\prime} j^{\prime}: i^{\prime \prime} j^{\prime \prime}} \psi_{i^{\prime \prime} j^{\prime \prime}}^{k}
$$

In matrix form it is written as

$$
P=R Q,
$$

where $P$ is the diagonal matrix whose diagonal entries consist of the $P_{i j}$ and $R=\left(R_{i j ; i^{\prime} j^{\prime}}\right), Q=\left(Q_{i j ; i^{\prime} j^{\prime}}\right)$ are $g ! \times g !$ matrices.

We shall construct a basis $\left\{\widehat{\psi}_{i j}^{k}\right\}$ of gr $M_{c}^{k}$ as a gr $\mathcal{D}_{g-k}$-module modifying $\left\{\psi_{i j}^{k}\right\}$ appropriately so that they satisfy

$$
\xi_{g-k} \widehat{\psi}_{i j}^{k} \in \sum_{i^{\prime} \leq i+1} \mathcal{K}\left[\xi_{1}, \ldots, \xi_{g-k-1}\right] \widehat{\psi}_{i^{\prime} j^{\prime}}^{k}+K^{k}
$$

To this end we use the relation 4.7. Let us write it more explicitly as

$$
\begin{aligned}
\varphi_{i j}= & \sum q_{i j ; i+1 j^{\prime}} \psi_{i+1, j^{\prime}}^{k}+\sum_{l=1}^{g-k} \sum_{j^{\prime}} q_{i j ; i j^{\prime}}^{l} \xi_{l} \psi_{i j^{\prime}}^{k} \\
& +\sum_{i^{\prime}<i,|\alpha|+i^{\prime}=i+1} q_{i j ; i^{\prime} j^{\prime}}^{\alpha} \xi^{\alpha} \psi_{i^{\prime} j^{\prime}}^{k} .
\end{aligned}
$$

For the sake of simplicity we identify $q_{i j ; i j^{\prime}}^{l}$ with $q_{i j ; i j^{\prime}}^{\alpha}, \alpha=(0, \ldots, 1, \ldots, 0)$, where 1 is in the $l$-th place.

We construct $\left\{\widehat{\psi}_{i j}^{k}\right\}$ satisfying 4.11 by induction on $i$. 
Let us consider the case $i=1$. Then 4.12 becomes

$$
\varphi_{11}=\sum q_{11 ; 2 j} \psi_{2 j}^{k}+\sum_{l=1}^{g-k} q_{11 ; 11}^{l} \xi_{l} \psi_{11}^{k}
$$

We proceed by considering several cases

(i) Assume $q_{11 ; 11}^{g-k} \neq 0$. In this case 4.11 holds for $(i, j)=(1,1)$ by defining $\widehat{\psi}_{11}^{k}=\psi_{11}^{k}$ and $\widehat{\psi}_{2 j}^{k}=\psi_{2 j}^{k}$.

(ii) Assume $q_{11 ; 11}^{g-k}=0$ and $q_{11 ; 2 j} \neq 0$ for some $j$. In this case we modify $\psi_{2 j}^{k}$ to $\widehat{\psi}_{2 j}^{k}=\psi_{2 j}^{k}-\xi_{g-k} \psi_{11}^{k}$. The operators $P_{i j}$ are changed correspondingly. We can take the product $P_{2 j} P_{11}$ as an operator which annihilates $\left.\widehat{\psi}_{2 j}^{k}\right|_{Y^{k+1}}$, since $P_{2 j} P_{11} \widehat{\psi}_{2 j}^{k} \in K^{k}$. We set $\widehat{\psi}_{2 j^{\prime}}^{k}=\psi_{2 j^{\prime}}^{k}$ for $j^{\prime} \neq j$ and $\widehat{\psi}_{11}^{k}=\psi_{11}^{k}$. Then 4.11 holds for $(i, j)=(1,1)$.

(iii) Assume $q_{11 ; 11}^{g-k}=0$ and $q_{11 ; 2 j}=0$ for all $j$. This is impossible. In fact, suppose that this is the case. We consider the equation 4.10 modulo the ideal

$$
I=\sum_{l=1}^{g-k-1}\left(\operatorname{gr} \mathcal{D}_{g-k}\right) \xi_{l}
$$

Then the right hand side is degenerate while the left hand side is non-degenerate.

On the whole we have constructed $\widehat{\psi}_{i j}^{k}, i \leq 2$, such that 4.11 holds for $(i, j)=(1,1)$.

Assume that $\widehat{\psi}_{i^{\prime} j^{\prime}}^{k}, i^{\prime} \leq i, 1 \leq j^{\prime} \leq r_{i^{\prime}}$, are constructed in such a way that 4.11 holds for $\left(i^{\prime}, j^{\prime}\right), i^{\prime}<i$, by solving 4.12. As a consequence of the change from $\left\{\psi_{i^{\prime} j^{\prime}}^{k} \mid i^{\prime} \leq i\right\}$ to $\left\{\widehat{\psi}_{i^{\prime} j^{\prime}}^{k} \mid i^{\prime} \leq i\right\}$ the matrices $P, Q, R$ may change. However the equation 4.10 still holds and $P$ remains non-degenerate. Therefore we use the same symbols $P, Q, R$ and their components in the argument below for the sake of simplicity.

Using the relation 4.11 for $\widehat{\psi}_{i^{\prime} j^{\prime}}^{k}$ for $i^{\prime}<i$, the formula 4.7 for $\varphi_{i j}$ can be written as

$\tilde{\varphi}_{i j}=\sum q_{i j ; i+1 j^{\prime}} \psi_{i+1, j^{\prime}}^{k}+\sum_{l=1}^{g-k} \sum_{j^{\prime}=1}^{r_{i}} \tilde{q}_{i j ; i j^{\prime}}^{l} \xi_{l} \widehat{\psi}_{i j^{\prime}}^{k}+\sum_{i^{\prime}<i,|\alpha|+i^{\prime}=i+1, \alpha_{g-k}=0} \tilde{q}_{i j ; i^{\prime} j^{\prime}}^{\alpha} \xi^{\alpha} \widehat{\psi}_{i^{\prime} j^{\prime}}^{k}$

for some $\tilde{\varphi}_{i j} \in K_{i+1}^{k}$ and some $\tilde{q}_{i j ; i^{\prime} j^{\prime}}^{\alpha}, i^{\prime} \leq i$. Here $q_{i j ; i^{\prime} j^{\prime}}^{\alpha}$ changes to $\tilde{q}_{i j ; i^{\prime} j^{\prime}}^{\alpha}$ as a consequence of the use of 4.11). Notice that the effect on the matrix $Q$ of applying the relation 4.11 is equivalent to making fundamental transformations on rows of $Q$, since 4.11 is obtained by solving 4.12 . 
We have

$$
\tilde{\varphi}_{i j}=\sum q_{i j ; i+1, j^{\prime}} \psi_{i+1, j^{\prime}}^{k}+\sum_{j^{\prime}=1}^{r_{i}} \tilde{q}_{i j ; i j^{\prime}}^{g-k} \xi_{g-k} \widehat{\psi}_{i j^{\prime}}^{k} \quad \bmod I \operatorname{gr} M_{c}^{k} .
$$

The rank of the $r_{i} \times\left(r_{i}+r_{i+1}\right)$ matrix

$$
\left(\left(q_{i j ; i+1, j^{\prime}}\right)_{1 \leq j^{\prime} \leq r_{i+1}},\left(\tilde{q}_{i j ; i j^{\prime}}^{g-k}\right)_{1 \leq j^{\prime} \leq r_{i}}\right)_{1 \leq j \leq r_{i}}
$$

is maximal, for otherwise $P$ is not non-degenerate. Thus, as in the case of $i=1$, modifying $\psi_{i+1, j^{\prime}}^{k}$ to $\widehat{\psi}_{i+1, j^{\prime}}^{k}=\psi_{i+1, j^{\prime}}^{k}-\xi_{g-k} \psi_{i j^{\prime \prime}}^{k}$ for some $j^{\prime}$ and $j^{\prime \prime}$ if necessary, we get $\left\{\widehat{\psi}_{i^{\prime}, j^{\prime}}^{k} \mid i^{\prime} \leq i+1\right\}$ such that 4.11 holds for $\left(i^{\prime}, j^{\prime}\right), i^{\prime} \leq i$. Notice that, in the last step $i=g, \tilde{q}_{g+1,1 ; g 1}^{g-k} \neq 0$ is automatic.

Thus a gr $\mathcal{D}_{g-k}$-free basis $\left\{\widehat{\psi}_{i j}^{k}\right\}$ of $\operatorname{gr} M_{c}^{k}$ satisfying the condition 4.11 is constructed. Set

$$
\psi_{i j}^{k+1}=\left.\widehat{\psi}_{i j}^{k}\right|_{Y^{k+1}}
$$

Then 4.11 implies that

$$
\xi_{g-k} \psi_{i j}^{k+1} \in \sum\left(\operatorname{gr} \mathcal{D}_{g-k-1}\right) \psi_{i^{\prime} j^{\prime}}^{k+1}
$$

Lemma 4.4. If $c+\sum_{i \in I} a_{i} \notin \Gamma$ for any subset $I$ of $\{1, \ldots, k\}$, the restriction map gr $\pi_{k}$ is surjective.

Proof. Use a similar argument to the proof of Lemma 4.3 .

By the lemma and the inductive assumption on $\operatorname{gr} M_{c}^{k}$, gr $M_{c}^{k+1}$ is generated by $\left\{\psi_{i j}^{k+1}\right\}$ over gr $\mathcal{D}_{g-k}$. Then 4.13 implies that

$$
\operatorname{gr} M_{c}^{k+1}=\sum\left(\operatorname{gr} \mathcal{D}_{g-k-1}\right) \psi_{i j}^{k+1} .
$$

It follows from (3.1) that the sum on the right hand side is a direct sum. This completes the proof of Proposition 4.1 .

\section{Acknowledgements}

This research is supported by Grant-in-Aid for Scientific Research (B) 17340048.

\section{References}

[K] I. M. Krichever, Methods of algebraic geometry in the theory of non-linear equations, Russian Math. Surveys 32 (1977), 185-213. Zbl 0386.35002 MR 0516323

[Mir] A. E. Mironov, Commutative rings of differential operators corresponding to multidimensional algebraic varieties, Siberian Math. J. 43 (2002), 888-898. Zbl 1006.14016 MR 1946267 
[M1] D. Mumford, Abelian varieties, Oxford Univ. Press, 1970. Zbl 0223.14022 MR 0282985

[M2] D. Mumford, Tata lectures on theta I, Birkhäuser, 1983. Zbl 1112.14002 MR 2352717

[N1] A. Nakayashiki, Structure of Baker-Akhiezer modules of principally polarized abelian varieties, Duke Math. J. 62 (1991), 315-358. Zbl 0732.14008 MR 1104527

[N2] Commuting partial differential operators and vector bundles over abelian varieties, Amer. J. Math. 116 (1994), 65-100. Zbl 0809.14016 MR 1262427

[R] M. Rothstein, Dynamics of the Krichever construction in several variables, J. Reine Angew. Math. 572 (2004), 111-138. Zbl 1142.37362 MR 2076122 